\title{
Detonation interaction with an interface
}

\author{
D. H. Lieberman ${ }^{\text {a) }}$ and J. E. Shepherd \\ Graduate Aeronautical Laboratories, California Institute of Technology, Pasadena, California 91125, USA
}

(Received 6 March 2007; accepted 28 June 2007; published online 18 September 2007)

Detonation interaction with an interface was investigated, where the interface separated a combustible from an oxidizing or inert mixture. The ethylene-oxygen combustible mixture had a fuel-rich composition to promote secondary combustion with the oxidizer in the turbulent mixing zone (TMZ) that resulted from the interaction. Sharp interfaces were created by using a nitro-cellulose membrane to separate the two mixtures. The membrane was mounted on a wood frame and inserted in the experimental test section at a $45^{\circ}$ angle to the bulk flow direction. The membrane was destroyed by the detonation wave. The interaction resulted in a transmitted and reflected wave at a node point similar to regular shock refraction. A detonation refraction analysis was carried out to compare with the measured shock angles. It was observed that the measured angle is consistently lower than the predicted value. An uncertainty analysis revealed possible explanations for this systematic variation pointing to factors such as the incident wave curvature and the role of the nitro-cellulose diaphragm. Analysis of the TMZ and Mach stem formed from the reflection of the transmitted shock wave off the solid boundary were carried out and found to justify the size and strength of these features as a function of the test gas composition. The role of secondary combustion in the TMZ was also investigated and found to have a small influence on the wave structure. () 2007 American Institute of Physics. [DOI: 10.1063/1.2768903]

\section{INTRODUCTION}

When a detonation wave propagating in a gaseous combustible mixture is confined by a noncombustible gas, a complex interaction results between the detonation and mixture interface. The details of this interaction, termed detonation refraction, are dependent on the mixture compositions, the relative geometry of the detonation and interface, and the characteristic thickness of the interface.

The interface, a composition gradient between the combustible and noncombustible mixtures, is classified by comparing the cell size of the detonation with the interface thickness. A sharp interface occurs when the detonation cell size is much greater than the interface thickness. Diffuse interfaces result when the cell size is comparable to or much less than the interface thickness. In this study we limit our discussion to sharp interfaces practically implemented using a nitro-cellulose membrane at a $45^{\circ}$ angle to the oncoming detonation wave.

Detonation propagation through an interface results in a complex interaction producing a transmitted and reflected wave as well as a contact surface that develops into a turbulent mixing zone (TMZ) as shown in Fig. 1. It is possible for subsequent combustion to occur in the TMZ by choosing a combustible mixture such that the combustion products are incompletely oxidized. This allows further reactions to take place if the mixture downstream of the interface contains an oxidizer. $^{1}$

There has been limited experimental research carried out in this area. Experiments ${ }^{2}$ were carried out using a methaneoxygen detonation that propagated through a nitro-cellulose

\footnotetext{
${ }^{a)}$ Present address: Exponent Failure Analysis Associates, Los Angeles, CA, USA. Electronic mail: dlieberman@exponent.com
}

interface at various angles to the oncoming flow. The test gas mixtures were less sensitive methane-oxygen mixtures or air. Other studies ${ }^{3,4}$ have investigated detonation diffraction between multiple combustible mixtures with varying sensitivity, separated in parallel channels. In these experiments, oblique detonation waves were observed to propagate in the less sensitive mixture.

There has been some previous work that included chemical reactions in a shock-polar analysis ${ }^{5,6}$ to investigate the role of ideal gas dissociation during shock wave refraction with an interface and multiple shock wave interaction. One more relevant work ${ }^{7}$ has modeled the deflection of a contact surface arising from a detonation propagating through a channel with a compressible, nonreacting boundary for the limiting case of the detonation velocity being normal to the interface.

The aim of the following study is to experimentally record the detonation refraction process and address the key physical features that result. A detonation refraction analysis is developed analytically as a basis of comparison to the experimental observations. Some of the main issues to be discussed are the geometry of the transmitted and reflected waves, the growth of the turbulent mixing zone, and formation of a Mach stem along the top boundary. The presence of secondary combustion between the partially oxidized detonation products and oxidizer in the TMZ is also examined.

\section{EXPERIMENTAL SETUP}

The experiments were carried out using the GALCIT detonation tube (GDT), ${ }^{8,9}$ which is $7.3 \mathrm{~m}$ long with an insidediameter of $280 \mathrm{~mm}$. It is connected to a $0.762 \mathrm{~m}$ long square test section with inside dimensions of $15 \mathrm{~cm}$ by 

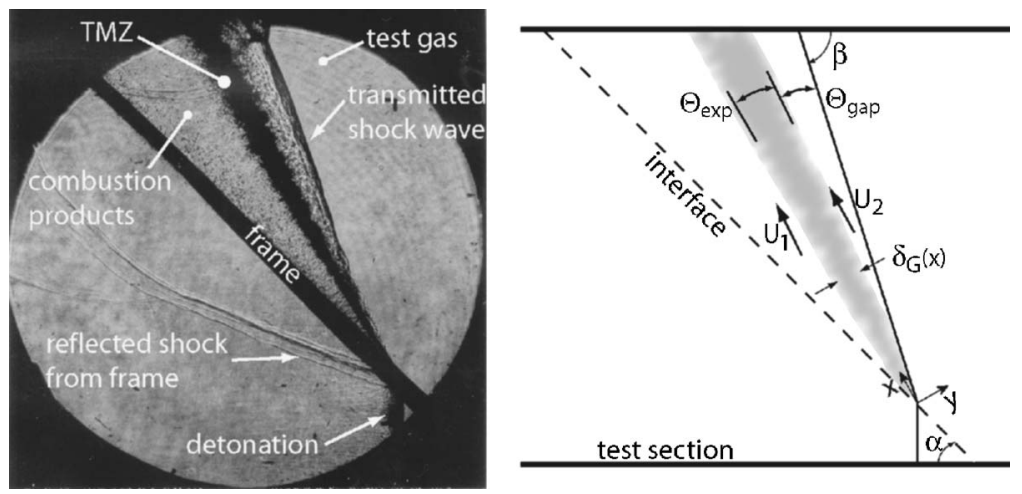

FIG. 1. A schlieren image of a detonation propagating from left to right through an interface (Exp. \#1922). The measured growth rate angle $\Theta_{\exp }$ and angle $\Theta_{\text {gap }}$ between the transmitted shock and TMZ are defined. Velocities $U_{1}$ and $U_{2}$ in the TMZ reference frame, and the shear layer thickness $\delta_{G}$, a function of $x$, are included.

$15 \mathrm{~cm}$. A wave cutting device extends $1 \mathrm{~m}$ into the end of the GDT to cut out a square section of the circular detonation front before entering the test section. A sliding valve assembly separates the ethylene-oxygen combustible mixture in the GDT from the oxidizer or inert diluent in the test section during the experimental preparation. A nitro-cellulose membrane, mounted on a wood frame is placed in the window of the test section.

Figure 2 is a view of the test section illustrating the location of the end flange of the GDT, the sliding valve assembly, and the test section. Visualization for the experiments, using a schlieren system, ${ }^{8}$ was made through an optical viewport that could be arranged in two separate positions. The first position is located $27.5 \mathrm{~cm}$ downstream of the sliding valve and is referred to as port 1 . The second viewport position is located $56 \mathrm{~cm}$ downstream of the sliding valve (Fig. 2) and is referred to as port 2. The locations of the pressure transducers and a quartz window on the end wall used for fluorescence imaging are also shown. Fuel rich ethylene-oxygen mixtures with equivalence ratios $\Phi=1$ and 2.5 occupied the GDT, and either oxygen, nitrogen, or nitrous oxide occupied the test section. The initial pressure and temperature for all experiments were $15 \mathrm{kPa}$ and $298 \mathrm{~K}$, respectively. Detonations were initiated in the GDT by discharging a $2 \mu \mathrm{F}$ bank of capacitors charged to $9 \mathrm{kV}$ through

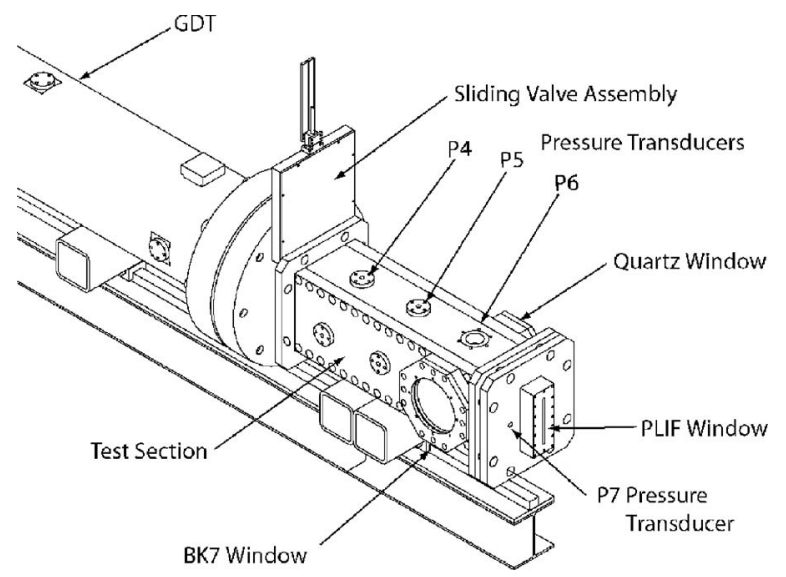

FIG. 2. A schematic of the test section with the sliding valve assembly and the end flange of the GDT. a $0.16 \mathrm{~mm}$ copper wire. Detonation velocities were measured to within $5 \%$ of the Chapman-Jouguet (CJ) speed. The cell sizes of the detonations were measured using the soot foil technique ${ }^{10}$ and were in all cases below $5 \mathrm{~mm}$.

Nitro-cellulose membranes were used to create a sharp interface between the combustible mixture and the oxidizer (or inert gas). The primary constituents of the membrane solution are nitro-cellulose and castor oil and was prepared in a fume hood. The membrane solution was spread out on a bed of water and allowed to dry. A rectangular wood frame made out of $3 \mathrm{~mm}$ thick and $9.5 \mathrm{~mm}$ wide cedar strips and coated with adhesive was placed on the dry membrane solution and then removed from the water surface. The frame was mounted in the GDT with two brackets to hold the frame in place. Nitro-cellulose membranes prepared in this fashion were measured to be on the order of 1 to $10 \mu \mathrm{m}$ thick. $^{11}$

In some cases the frame would be slightly warped when prepared, and when mounted in the test section, it would then further change shape slightly. Given the extreme fragility of the membrane, it was impossible to correct for this and it was difficult to measure the extent of warping due to the nature of the installation of the membrane.

Difficulties in the filling procedure arise because of the use of a nitro-cellulose membrane. It is necessary to fill simultaneously on both sides of the membrane to avoid tearing it. ${ }^{12}$ In the current setup, the additional complexity is that the combustible mixture needs to be mixed once it is loaded into the GDT. The filling is accomplished by closing the sliding valve to separate the test section from the GDT. The GDT is then filled with the combustible mixture and mixed, and then the test section is filled with the test gas. At the end of this procedure the test gas is on both sides of the nitro-cellulose membrane situated at port 1 .

Replacing the slug of test gas mixture between the sliding valve and membrane with the combustible mixture in the GDT is accomplished by opening the sliding valve and allowing the gravity current to sweep out and mix with the test gas. The technique is based on observations made during prior experiments. ${ }^{13}$ The sliding valve is left open for $2 \mathrm{~min}$, and then the fire button is pressed.

The technique of using a gravity current to displace and mix with the remaining slug of test gas mixture was validated by propagating a detonation into the test section without an interface. A slightly nonplanar detonation observed 


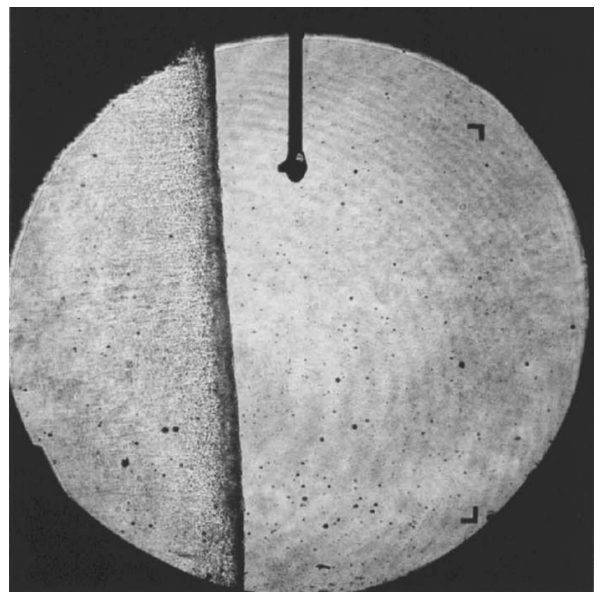

FIG. 3. A schlieren image of a detonation propagating from left to right corresponding to Exp. \#1903. The sting entering from the top is a remnant from a previous experiment.

during these tests is shown in Fig. 3 with a detonation velocity within $10 \%$ of the predicted Chapman-Jouguet value. The volume of test gas initially between the sliding valve and the membrane represents $1.3 \%$ of the GDT volume.

\section{DETONATION REFRACTION THEORY}

Consider a detonation wave propagating through sharp interfaces at an oblique angle (Fig. 1). We analytically determine the strength of the transmitted and reflected waves as well as the thermodynamic states resulting from the interaction to compare with experimental results. Two limiting cases are considered. First, the interaction is sufficiently far from the interaction point (node) such that the combustion products are in chemical equilibrium. Second, the interaction is considered sufficiently near the node, i.e., much smaller than the induction zone length, such that no chemical reactions have occurred and the composition can be considered frozen but the gas is otherwise in thermal equilibrium.

The Zeldovich, von Neumann, and Doering (ZND) model is used to quantify the length scales associated with the inner and outer solutions. The induction length is the critical length scale that separates the chemical equilibrium (outer) and frozen (inner) regions. For the mixtures in this study, corresponding to an ethylene-oxygen detonation at $\Phi=2.5$ and $15 \mathrm{kPa}$ initial pressure, the induction length, $\Delta$ $=0.06 \mathrm{~mm}$, and the solution is close to chemical equilibrium at $\Delta_{\text {equil }}=0.4 \mathrm{~mm}$. The reaction zone thickness is defined as the distance behind the incident shock at which the temperature approaches $1 \%$ of its chemical equilibrium value. By definition, the inner and outer region scales are related to the reaction zone and equilibrium lengths by

$$
\Delta_{\text {outer }} \gg \Delta_{\text {equil }}>\Delta \gg \Delta_{\text {inner }} \text {. }
$$

The submillimeter induction zone length indicates that we will not be able to observe the frozen region in these experiments. No further discussion is given here of this case.

The problem of shock wave refraction can be classified by the "refractive" index ${ }^{14}$ at the contact discontinuity, defined by the ratio of the incident wave to the transmitted

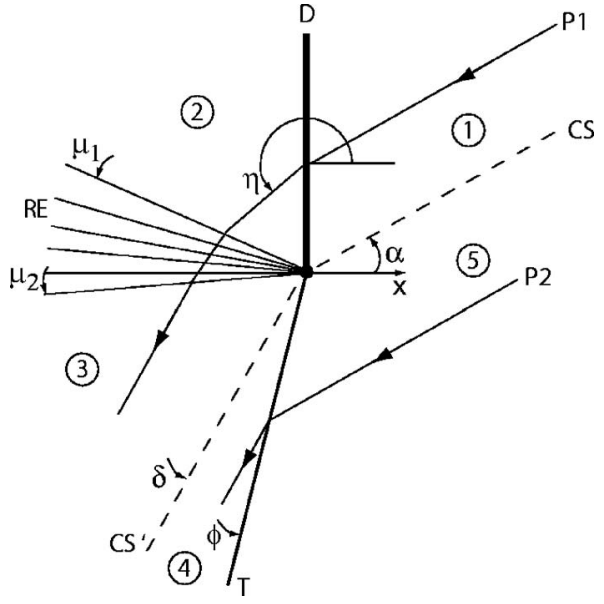

FIG. 4. Detonation refraction configuration and labeling showing states, particle paths, and wave angles.

wave velocities $U_{i}$ and $U_{t}$, respectively. If $U_{i}<U_{t}$, then the refraction is considered slow-fast. On the other hand if $U_{i}>U_{t}$, the refraction is considered fast-slow. If $U_{i}=U_{t}$ there is no refraction. For a given refractive index there are two types of wave systems: regular and irregular that are separated by an interface angle denoted the critical angle. Regular refraction consists of only waves that are straight and intersect at a single point called the node. Irregular refraction encompasses all scenarios that are not regular. Typically, irregular refraction exhibits added features such as Mach reflections and precursor shock waves. The type of reflected wave (expansion or shock) depends on the state of the detonation products (region 2, Fig. 4), the test gas composition, and the angle ( $\alpha$, Fig. 4$)$ between the detonation wave and the interface. This is most easily visualized on a pressure versus deflection plot. If the pressure-deflection locus for region 2 is situated inside the transmitted shock polar, a reflected expansion wave occurs. If the pressure-deflection locus for region 2 is situated outside the transmitted shock polar, a reflected shock wave occurs. Calculations with various fuel-oxidizer mixtures and test gases were carried out and determined that both solution types are physically possible. For example, if a detonation wave propagates from a lean hydrogen-oxygen mixture into a pure nitrous oxide test gas, a reflected shock wave will result.

\section{A. Analytical description of equilibrium region}

The following analysis considers a detonation wave $D$, propagating through a contact surface $\mathrm{CS}_{1}$ that is inclined at an angle $\alpha$ to the wave propagation direction shown in Fig. 4. The resulting transmitted wave $T$, reflected expansion and contact surface $\mathrm{CS}_{2}$ are illustrated for a typical interaction. The analysis, carried out in the reference frame of the node, has five uniform regions, labeled 1-5. Region 1 corresponds to the combustible gas and region 5 to the inert or oxidizing test gas. These regions contain unprocessed gas still in its initial state. Traversing the domain counterclockwise, regions 1 and 2 are separated by the detonation wave and region 2 contains equilibrium detonation products. The gas from region 2 then expands through the reflected wave, 


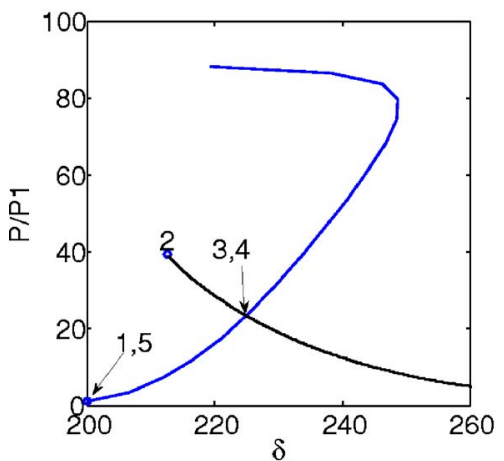

(a)

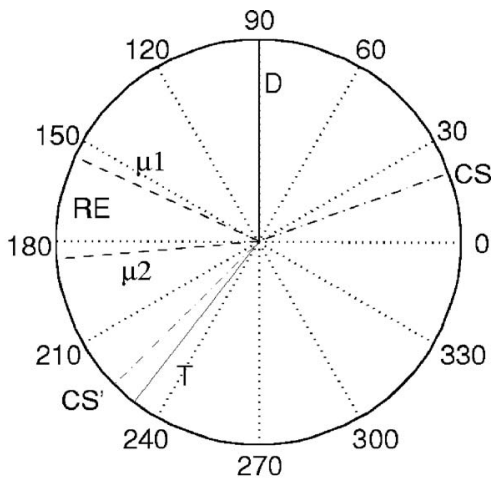

(b)
FIG. 5. Graphical solution of an ethylene-oxygen detonation with equivalence ratio of 2.5 propagating into oxygen with contact surface $\alpha=20$ and initial temperature and pressure of $295 \mathrm{~K}$ and $15 \mathrm{kPa}$, respectively. (a) Pressure vs flow deflection. (b) Polar plot indicating the wave structure. shown as an expansion, and reaches a new state in region 3. Region 4 contains gas from region 5 that has been processed by the transmitted shock. Particle paths P1 and P2 illustrate the trajectory of gas through the system of waves with the angle $\eta$ - $\alpha$ measuring the deflection of $\mathrm{P} 1$ across the detonation wave. The angles of the waves are all referenced to the axis labeled $x$ and measured in the counterclockwise direction. The initial orientation of the contact surface or interface is $\alpha$; the complement of $\alpha$ is the angle between the detonation and contact surface. The angles $\mu_{1}$ and $\mu_{2}$ denote the orientations of leading and trailing characteristics of the reflected expansion, respectively, while $\delta$ and $\phi$ define the contact surface deflection and transmitted shock wave angles, respectively.

The analysis assumes that the detonation is a discontinuity with the state of the products determined by the Chapman-Jouguet (CJ) detonation theory. The shock waves are also considered as discontinuities. The thermodynamic state of a mixture passing from one state to another is determined by equilibrium chemistry computed with a program based on the Cantera chemical equilibrium software. Diffusional and viscous effects as well as body forces are omitted from this study. The program first determines the specific angle where no reflected wave occurs, i.e., $\alpha^{*}$, and then imposes an expansion wave or shock wave as the reflected wave. The angle $\alpha^{*}$ at which no reflected wave results is determined by a separate analysis ${ }^{13}$ and, for the mixtures in regions 1 and 5 of interest in this study, occur at $\alpha=\alpha^{*}$ $\approx 88^{\circ}$. When $\alpha<88^{\circ}$, a reflected expansion results.

\section{Geometrical relations}

In the reference frame of the node, we consider that the unreacted mixture in region 1 and inert or oxidizer gas in region 5 are moving with velocities

$$
\begin{aligned}
& \vec{w}_{1}=-V_{\mathrm{CJ}} \hat{i}-V_{\mathrm{CJ}} \tan \alpha \hat{j}, \\
& \vec{w}_{5}=\vec{w}_{1},
\end{aligned}
$$

where the detonation velocity is denoted by $V_{\mathrm{CJ}}$. The directions of the velocities are tangent to the particle paths P1 and P2, depicted in Fig. 4(a). The velocity in region 2 is determined by the post-detonation conditions and is given as

$$
\vec{w}_{2}=-c_{2} \hat{i}-V_{\mathrm{CJ}} \tan \alpha \hat{j},
$$

$$
\eta=\tan ^{-1}\left(\frac{c_{2} \tan \alpha}{V_{\mathrm{CJ}}}\right)+\pi,
$$

where $\vec{w}_{2}$ is the velocity of the detonation products moving at an angle $\eta$, and $c_{2}$ is the sound speed in region 2 . The velocities $\vec{w}_{3}=f n\left(\delta_{3}-\eta, \alpha\right)$ and $\vec{w}_{4}=f n(\phi, \alpha)$ are expressed as functions of the flow deflection angles $\delta_{3}$ and $\delta_{4}$, the transmitted shock angle $\phi$, contact surface angle $\alpha$, and the thermodynamic states of regions 2 and 5 .

\section{Governing equations and matching conditions}

The geometric relations in Sec. III A 1 are used in conjunction with the conservation equations, equation of state, and matching conditions to uniquely determine the wave structure and thermodynamic states of the system. To treat oblique shock waves, the one-dimensional shock jump conditions must be solved iteratively since the enthalpy $h$ of an equilibrium ideal gas mixture is a nonlinear function of temperature and pressure. The subscripts $n$ and $v$ denote the velocity components normal and perpendicular to the transmitted shock wave, respectively. The local pressure, density, and velocity are denoted by $p, \rho$, and $w$, respectively. The flow deflection angle $\Delta \theta_{E}$, resulting from the reflected expansion fan is obtained by integrating

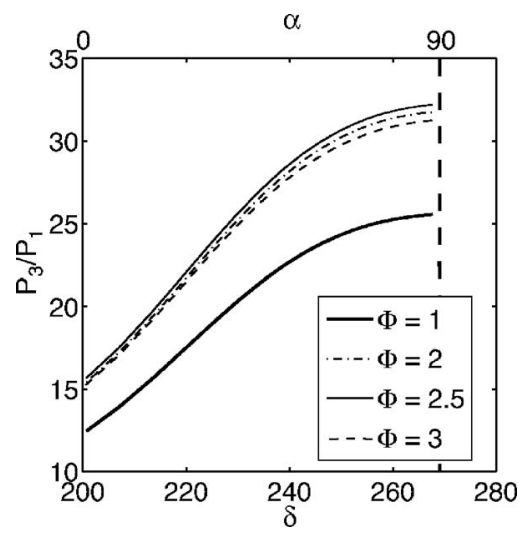

FIG. 6. Normalized pressure vs flow deflection plot showing the locus of solutions to regions 3 and 4 for $\alpha=[0,90]$. Region 1: Ethylene-oxygen with $\Phi=1,2,2.5,3$. Region 2: Molecular oxygen. The initial pressure and temperature are $15 \mathrm{kPa}$ and $295 \mathrm{~K}$, respectively. 


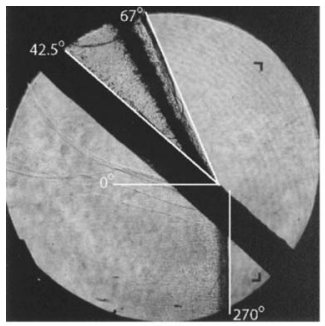

(a)

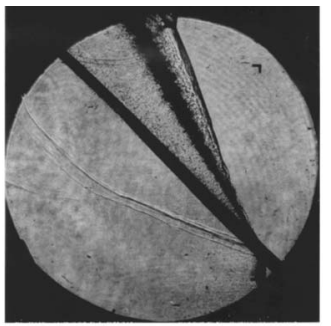

(b)

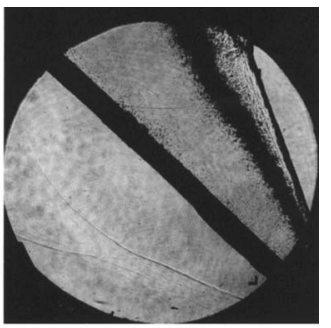

(c)

$$
d \theta_{E}=\frac{\sqrt{\mathrm{Ma}^{2}-1}}{1+(\Gamma-1) \mathrm{Ma}^{2}} \frac{d \mathrm{Ma}}{\mathrm{Ma}}
$$

where $\Gamma$ is the fundamental gas derivative and $\mathrm{Ma}$ is the Mach number. $\Gamma$ is defined as ${ }^{15}$

$$
\Gamma \equiv \frac{c^{4}}{2 v^{3}}\left(\frac{\partial^{2} v}{\partial P^{2}}\right)_{s}=\frac{1}{c}\left(\frac{\partial(\rho c)}{\partial \rho}\right)_{s}
$$

with subscript $s$ denoting a path with constant entropy. To evaluate (6) at a given state a script was written that calculated the finite difference approximation to the derivative by perturbing the initial density by $0.1 \%$ and then equilibrating with constant entropy and density to determine the new state. For a perfect gas, the fundamental gas derivative reduces to $\Gamma=(\gamma+1) / 2$.

Pressure and flow deflection must match at the interface between regions 3 and 4 , a requirement that closes the problem. ${ }^{15}$ This sets up a system of nonlinear algebraic equations

$$
\begin{aligned}
& \delta_{3}\left(\Delta \theta_{E}, \alpha, S_{1}\right)=\delta_{4}\left(\phi, \alpha, S_{5}\right), \\
& P_{3}\left(\Delta \theta_{E}, \alpha, S_{1}\right)=P_{4}\left(\phi, \alpha, S_{5}\right),
\end{aligned}
$$

relating the transmitted shock angle and the flow deflection through the reflected expansion fan. The flow deflection angle between region 2 and region 3 is defined as $\Delta \theta_{E} \equiv \delta_{3}$ $-\eta$, and $S_{1}$ and $S_{5}$ refer to the thermodynamic states in these regions. Figure 5(a) is the shock polar graphical solution for a contact surface angle $\alpha=20^{\circ}$. The mixture in region 1 is ethylene-oxygen with equivalence ratio of 2.5 and in region 5 is molecular oxygen. The initial temperature and pressure of regions 1 and 5 are $295 \mathrm{~K}$ and $15 \mathrm{kPa}$, respectively. The thermodynamic state of region 2 is determined by the $\mathrm{CJ}$ detonation solution. If we considered the possibility of overdriven detonations, there would exist a locus of end states for region 2 on the pressure flow deflection plane; ${ }^{9}$ however, this is not shown in Fig. 5(a). The corresponding wave structure is shown in Fig. 5(b) on a polar plot to highlight the wave angles. The expansion fan is bounded by the two Mach waves, $\mu_{1}$ and $\mu_{2}$, of regions 2 and 3 , respectively. The deflected contact surface $\mathrm{CS}^{\prime}$ separating regions 3 and 4 has a calculated flow deflection angle of $225^{\circ}$.

For a given $\alpha$, there is a unique geometrical wave solution with a specified $\delta_{i}$ and $P_{i}$ with subscript $i$ denoting region 3 or 4 . Figure 6 plots the pressure $P_{i}$ normalized by the pressure in region 1 versus flow deflection angle $\delta_{i}$ for angles of $0<\alpha<90$. The lines refer to solutions with ethyleneoxygen mixtures with equivalence ratios of $1,2,2.5$, and 3 . The initial temperature and pressure for regions 1 and 5 are $295 \mathrm{~K}$ and $15 \mathrm{kPa}$, respectively. Molecular oxygen is the gas that comprises region 5 .

TABLE I. Table of transmitted shock angle $\beta$, angle $\Theta_{\text {exp }}$, and transmitted-shock-TMZ angle $\Theta_{\text {gap }}$ with ethylene-oxygen mixtures, with equivalence ratio $\Phi$, and oxygen, nitrogen, or nitrous oxide as the test gas. Subscript "exp" denotes an experimental measurement.

\begin{tabular}{llllllll}
\hline \hline Shot \# & $\Phi$ & Test gas & $\alpha_{\text {exp }}$ & $\beta$ & $\beta_{\text {exp }}$ & $\Theta_{\text {exp }}$ & $\Theta_{\text {gap }}$ \\
\hline 1906 & 2.5 & $\mathrm{~N}_{2}$ & 44 & 72.1 & 69 & 4.5 & 6 \\
1907 & 2.5 & $\mathrm{~N}_{2}$ & 41 & 70.4 & 67 & 6.5 & 3.5 \\
1912 & 2.5 & $\mathrm{O}_{2}$ & 45 & 71.4 & 67 & 6 & 6 \\
1914 & 2.5 & $\mathrm{O}_{2}$ & 43 & 70.2 & 63 & 6 & 3 \\
1915 & 2.5 & $\mathrm{O}_{2}$ & 44 & 70.8 & 67 & 4 & 5 \\
1916 & 2.5 & $\mathrm{~N}_{2} \mathrm{O}$ & 44 & 67.1 & 62 & 5 & 3 \\
1917 & 2.5 & $\mathrm{~N}_{2} \mathrm{O}$ & 44 & 67.1 & 65 & 5 & 2 \\
1919 & 1 & $\mathrm{~N}_{2} \mathrm{O}$ & 45 & 68.3 & 65 & 6.2 & 2 \\
1920 & 1 & $\mathrm{~N}_{2} \mathrm{O}$ & 44 & 67.7 & 65 & 6 & 3 \\
1921 & 1 & $\mathrm{~N}_{2} \mathrm{O}$ & 44 & 67.7 & 65 & 8.5 & 2 \\
1922 & 2.5 & $\mathrm{~N}_{2}$ & 45 & 72.7 & 70 & 7 & 7 \\
1923 & 1 & $\mathrm{O}_{2}$ & 45 & 72.1 & 65 & 4.8 & 5 \\
1925 & 1 & $\mathrm{O}_{2}$ & 44 & 71.6 & 67 & 4 & 5 \\
1926 & 1 & $\mathrm{O}_{2}$ & 45 & 72.1 & & 67 & 4.5 \\
1927 & 1 & $\mathrm{O}_{2}$ & 45 & 72.1 & 6.5 & 6 \\
\hline \hline
\end{tabular}


For the limiting case when $\alpha=0^{\circ}$, the solution is identical to the case studied by Dabora. ${ }^{7}$ The detonation products in the node fixed frame travel at the speed of sound of the burned products causing the angle of the expansion fan head to coincide with the detonation wave. In the limit of $\alpha$ $=90^{\circ}$, the interface becomes parallel to the detonation wave. Although this is a nonphysical solution, the result indicates that the detonation wave never reaches the contact surface and continuously propagates in the combustible mixture. The deflection angle approaches $270^{\circ}$ as the inflow velocity goes to infinity, which compacts the reflected and transmitted waves together.

The detonation refraction analysis will be compared to the experimentally observed refraction angles. In addition, this analysis will serve as a starting point to examine the growth of the turbulent mixing zone at the deflected contact surface $\mathrm{CS}^{\prime}$. Specifically, the thermodynamic states and velocities in regions 3 and 4 can be used to estimate shear layer growth (Sec. IV B).

\section{DETONATION REFRACTION EXPERIMENTS}

The key features in the detonation refraction experiments are shown in Fig. 1. The schlieren image shows a detonation propagating from left to right in a $\Phi=2.5$ ethylene-oxygen mixture through a nitro-cellulose membrane interface mounted on a wood frame into nitrogen. The membrane was glued onto the right-facing surface as viewed in all the figures showing the detonation-interface interaction. A transmitted shock wave and a turbulent mixing zone result from this interaction. There is also a reflected shock wave that originates from the wood frame. Detonation refraction theory predicts a weak reflected expansion for this interfacedetonation configuration. One additional flow feature is a small Mach stem located along the top wall at the end of the transmitted wave. Small particles are visible around the TMZ; these are remnants of the membrane.

The experiment is sufficiently repeatable so that it is possible to construct a time sequence with the schlieren images. Figure 7 shows three images corresponding to experiments \#1907, \#1922, and \#1906. These experiments use a $\Phi=2.5$ ethylene-oxygen combustible mixture and nitrogen as the test gas. The varying image capture times relative to image (a) are (b) $8 \mu \mathrm{s}$, and (c) $27 \mu \mathrm{s}$. It is observed that as the detonation moves from left to right the node, or point of origin of the transmitted shock, and TMZ moves progressively down the interface. The difference in frame size in Fig. 7 is due to frames (a) and (c) being constructed using $9 \mathrm{~mm}$ thick wood strips versus frame (b), which used $3 \mathrm{~mm}$
TABLE II. Possible sources of error addressing the discrepancy between the measured and predicted transmitted shock angle.

\begin{tabular}{lc}
\hline \hline Source of error & Uncertainty \\
\hline Nonplanar detonation wave & $\pm 4^{\circ}$ \\
Measurement error & $\pm 1^{\circ}$ \\
Warped wood frame & $\pm 2^{\circ}$ \\
Nonideal contact surface & $\pm 2^{\circ}$ \\
Schlieren light beam off axis & negligible \\
Schlieren stigmatism & \\
Curved transmitted shock wave & \\
Membrane fragments & \\
\hline \hline
\end{tabular}

thick wood strips. Additionally strips of tape were placed along the edges of the frame to seal any gaps that might exist between the frame and the test section walls.

In the present study, the ethylene-oxygen equivalence ratio and test gas mixture were varied. The results are quantified by measuring the angles of the transmitted shock wave, shear layer, and the gap between the two of them (Fig. 1).

The angles of the various features are measured and compared with the detonation refraction theory. The geometry of the detonation refraction waves in Sec. III is the mirror image of the experiment reflected along the horizontal axis, and is related by subtracting $180^{\circ}$ from the computed angles. A typical example is shown in Fig. 7(a). The interface angle is measured to be $42.5^{\circ}$ from the horizontal and the detonation wave is perpendicular to the horizontal axis and the transmitted shock wave is measured to be at an angle of $67^{\circ} \pm 1^{\circ}$. For this experiment, the detonation refraction theory predicts a transmitted wave angle of $70.4^{\circ}$. The resulting difference between experiment and theory is $6 \%$. Theory predicts that the contact surface angle should be $66^{\circ}$; however it does not factor in the growth of the mixing zone. In this experiment the TMZ is bounded between $60^{\circ}$ and $64^{\circ}$.

The measured transmitted shock angles reported in Table I are systematically lower than the predicted values by an average value of $4^{\circ}$. A list of possible errors are shown in Table II. The magnitudes of the uncertainties, measured in degrees are reported if estimated. The largest source of error results from a nonplanar detonation wave a product of the gas filling procedure used to sweep out the test gas. Other sources of error are from the warped wood frame and measuring the angles. The assumption that the deflected interface is treated as a contact surface and does not result in a shear layer also contributes to the error. The mixing-layers' displacement thickness was shown to be a function of heat

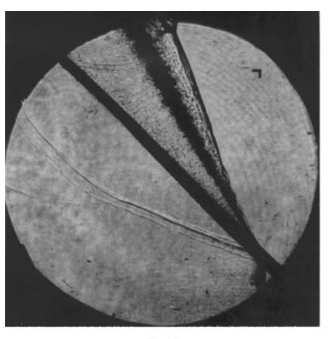

(a)

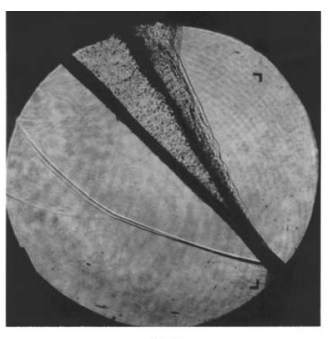

(b)

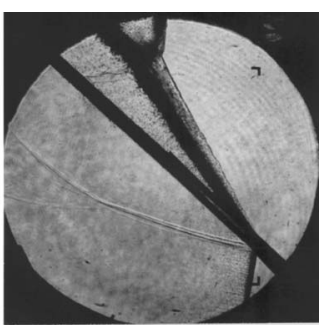

(c)
FIG. 8. A set of schlieren images showing Mach stems for different test gases. The interface is at $45^{\circ}$ with ethylene-oxygen $(\Phi=2.5)$ on the left side and (a) nitrogen (Exp. \#1922), (b) oxygen (Exp. \#1915), and (c) nitrous oxide (Exp. \#1916) on the right side of the interface. 


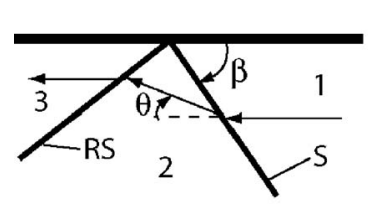

regular reflection

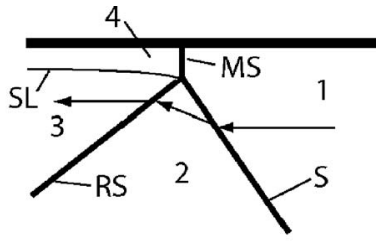

Mach-reflection
FIG. 9. A sketch of regular and Mach reflection with states 1, 2, and 3 and the incident (S) and reflected shocks (RS) labeled. The flow behind the Mach stem (MS) is subsonic with a slip line (SL) separating states 3 and 4.

release, ${ }^{16}$ which modifies the matching conditions and thus the wave structure.

One important observation is that in the region close to the node where the transmitted shock and TMZ coalesce, the wave curves and tends to a smaller angle $\beta$. This is observed in Figs. 7(a) and 7(b) where the detonation, TMZ, interface, and transmitted shock do not all meet at one point. Instead there is the intersection between the transmitted shock, TMZ, and frame and an intersection between the frame and detonation. The diaphragm or complex detonation-interface interaction in the vicinity of the nitro-cellulose membrane might be in part responsible for this behavior.

The formation of the Mach stem and growth of the turbulent mixing zone are two features that are explored in Secs. IV A and IV B, respectively. The shear layer is characterized by the growth rate $\Theta_{\text {model }}$, which is related to the local shear layer thickness $\delta_{G}(x)$ by

$$
\Theta_{\text {model }}=\tan ^{-1}\left(\frac{\delta_{G}(x)}{x}\right)
$$

The measured values $\Theta_{\text {exp }}$ are listed in Table I along with the gap angles $\Theta_{\text {gap }}$.

\section{A. Mach reflection}

The Mach stem was observed to depend on the type of test gas. Figure 8 shows three Mach stems with (a) nitrogen, (b) oxygen, and (c) nitrous oxide. The overall size and thickness increases from image (a) to (c). The role of the test gas on Mach stem formation is investigated to determine what role if any, secondary combustion in the TMZ plays in effecting the Mach stem size.

The condition for the onset of Mach reflection ${ }^{17}$ is calculated for the gas moving from state 2 to state 3 (Fig. 9). It is necessary to carry out the analysis in a convective frame of reference coinciding with the point of intersection between the incident shock wave and the wall of the regular reflection. The incident shock Mach number and angle $\beta$ are specified from the experimental conditions and determine the fluid state 2 that is deflected toward the solid boundary. This analysis calculates the maximum angle the flow can turn from state 2 to state 3 with a reflected shock wave. If the angle is less than the incident deflection angle then Mach reflection results. In the case of a perfect gas the oblique shock equation
TABLE III. Table of flow deflection angle from state 1 to state $2(\theta)$ and maximum flow deflection angle from state 2 to state $3\left(\theta_{\max }\right)$ resulting from a regular reflection with $\mathrm{Ma}_{2}$ referring to the Mach number in state 2 .

\begin{tabular}{lllcccc}
\hline \hline Test gas & $\Phi$ & $\alpha$ & $\theta$ & $\theta_{\max }$ & $\beta_{\exp }$ & $\mathrm{Ma}_{2}$ \\
\hline $\mathrm{N}_{2} \mathrm{O}$ & 2.5 & 45 & 49.3 & 7.45 & 65 & 1.30 \\
$\mathrm{~N}_{2} \mathrm{O}$ & 1 & 45 & 47.5 & 5.53 & 65 & 1.24 \\
$\mathrm{~N}_{2}$ & 2.5 & 42 & 41.5 & 0.25 & 66 & 1.03 \\
$\mathrm{~N}_{2}$ & 1 & 45 & 38.2 & & 66 & 0.98 \\
$\mathrm{O}_{2}$ & 2.5 & 45 & 41.7 & 1.05 & 65 & 1.08 \\
$\mathrm{O}_{2}$ & 1 & 45 & 39.5 & 0.43 & 65 & 1.04 \\
\hline \hline
\end{tabular}

$$
\tan \theta=2 \cot \beta \frac{\mathrm{Ma}^{2} \sin ^{2} \beta-1}{\mathrm{Ma}^{2}(\gamma+\cos 2 \beta)+2},
$$

which relates the flow turning angle $\theta$, to the incident Mach number $\mathrm{Ma}$, and the oblique shock angle $\beta$, is maximized at fixed Mach number. The critical value of $\beta$ is calculated for a given value $\mathrm{Ma}$, which is then substituted into Eq. (9) to result in a maximum flow deflection angle $\Theta_{\max }$. Table III lists the flow deflections after the incident oblique shock and the maximum turning angle possible using a reflected shock. In all cases, the Mach number $\mathrm{Ma}_{2}$ of the fluid after the incident shock is too small to generate the reflected shock needed to turn the parallel to the wall. Therefore, the regular solution is not possible and corroborates the appearance of a Mach stem. In some cases the post-incident-shock Mach number is less than $1 \mathrm{a}$, which guarantees that a reflected shock cannot occur and therefore there is no value for $\Theta_{\mathrm{mx}}$. The convective velocity at the intersection of the incident shock and the top wall is determined using the geometrical construction shown in Fig. 10. The detonation-shockinterface structure is compared in two positions to determine the relationship between the detonation velocity and oblique shock velocity

$$
U=V_{\mathrm{CJ}}\left(1-\frac{\tan \alpha}{\tan \beta}\right) \text {. }
$$

An oblique shock pressure-deflection graph is shown in Fig. 11(a) based on the regular reflection configuration (Fig. 9). The incident flow velocity is fixed at $U=1399 \mathrm{~m} / \mathrm{s}$ as determined by Eq. (10) for the case of $\alpha=45^{\circ}$ and $\beta=65^{\circ}$

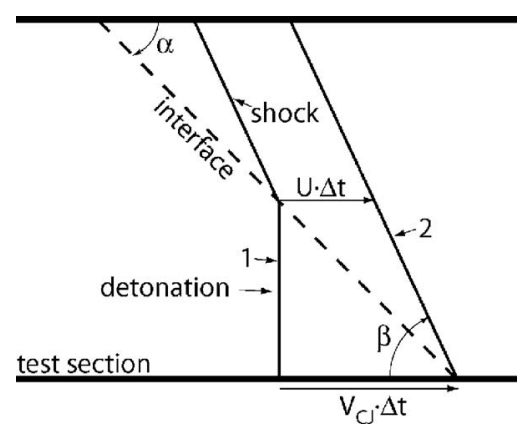

FIG. 10. A schematic of the detonation-interface interaction at two instants in time. The detonation and transmitted shock (1), and the fully emerged transmitted shock (2) are used to determine the shock velocity $U$. The interface angle and shock angle are denoted by $\alpha$ and $\beta$, respectively. 


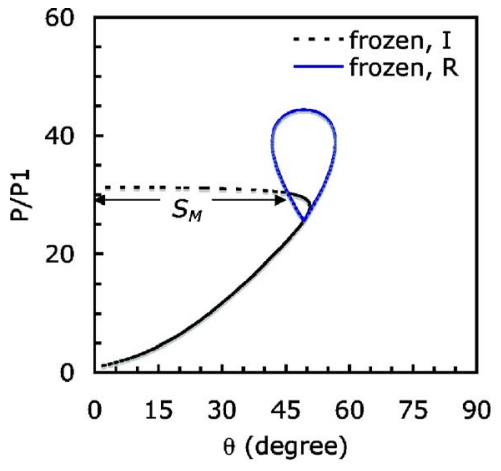

(a)

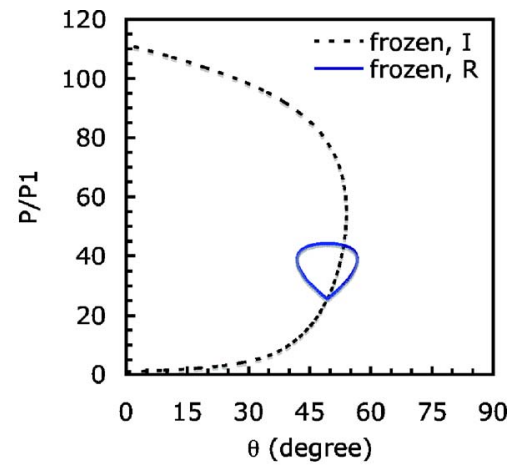

(b)
FIG. 11. Oblique shock polar normalized pressure $P / P_{1}$ as a function of flow deflection angle $\theta$ for incident $(I)$ and reflected $(R)$ waves in nitrous oxide. (a) The inflow velocity $U=1399 \mathrm{~m} / \mathrm{s}$, and (b) the inflow velocity $U$ is determined by Eq. (10) and is a function of $\alpha$. using nitrous oxide corresponding to the experimental conditions of shot \#1916. The reflected shock $(\mathrm{R})$ solution is also plotted. Graphically the reflected shock polar must cross the vertical axis for a regular reflection to occur. As this is not the case, a Mach stem forms to produce post-shock states over the range $S_{\mathrm{Ma}}$ occupying the region between the vertical axis and the intersection point between the reflected and incident shock polars. ${ }^{17}$

It is also interesting to plot the nonstandard shock polar obtained when the inflow velocity $U$ is constrained by the angle $\beta$ in Eq. (10). In this configuration, the locus of solutions for the incident pressure-deflection map is solely a function of $\alpha$ since detonation refraction theory determines the value of $\beta$ as a function of $\alpha$. The shock polar shows the possible states of the experiment. The reflected shock polar is determined the same way as in Fig. 11(a), by fixing the Mach number in state 2 and varying the shock angle $\beta$.

The Mach reflection discussion to this point has considered the composition to be frozen. Including the effect of dissociation and chemical reaction has been shown ${ }^{18}$ to either enhance or weaken the turning potential of the oblique shock. Generally, endothermic reactions result in larger flow deflection angles, while exothermic reactions result in smaller flow deflection angles. Of the test gases used in this study, oxygen and nitrogen are endothermic while nitrous oxide is exothermic. ${ }^{13}$

For the shock Mach numbers dealt with in this study $(\mathrm{Ma}<9)$ both oxygen and nitrogen are treated using frozen composition. Equilibrium analysis using of the decomposition of nitrogen and oxygen behind shock waves indicated that for shock Mach numbers below nine, less than 5\% of molecular oxygen or nitrogen dissociates. However, nitrous oxide readily decomposes exothermically at these conditions.

Detailed chemical reaction calculations are carried out to resolve the decomposition of nitrous oxide behind the shock wave. The calculations were carried out using Cantera solving the same equations used to determine the ZND solution with the difference that the incident shock velocity is imposed instead of the CJ condition. Equilibrium is reached as $t \rightarrow \infty$ when the flow conditions reach a steady value. Practically the time limit of the calculation was set to $1000 \mathrm{~s}$. The calculations used the GRI3.0 reaction mechanism validated for use in nitrous oxide detonations. The distance (or time) behind the shock at which equilibrium is reached decreases with increasing Mach number, a consequence of the Arrhen- ius dependent kinetics. To determine when equilibrium and frozen chemistry simplifications are valid it is important to compare the residence time of a convected fluid particle $\tau_{r}$ to the reaction time scale, defined as the time to attain $90 \%$ of the peak thermicity. The comparison is made in Fig. 12, where the reaction time is plotted as a function of Mach number for the exothermic decomposition of nitrous oxide. The fluid residence time scale was calculated to be $\tau_{r} \approx 62 \mu \mathrm{s}$ based on the detonation traveling $152.4 \mathrm{~mm}$, the length of the wood frame. For the equilibrium regime it is necessary that the reaction time be much less than the convective time. In this study two orders of magnitude are imposed to separate the frozen from the equilibrium regions. These regions are shown as bounded by the lines $\tau_{\mathrm{fr}}$ and $\tau_{\mathrm{eq}}$ in Fig. 12. The Mach numbers corresponding to the frozen and equilibrium boundaries are $\mathrm{Ma}=6$ and $\mathrm{Ma}=8$, respectively. Between these two bounds, the transient behavior depends on the details of the chemical reaction process.

For Mach numbers greater than eight the decomposition of nitrous oxide occurs sufficiently fast compared to the motion of the fluid through the field of view such that the decomposition region can be considered thin compared to the other length scales in the experiment.

Ultimately, the point of the equilibrium calculations is to show what effect they have on the flow deflection and thus the presence of a Mach stem. The goal is to resolve the observable differences in Mach stem size between the nitrous oxide and oxygen or nitrogen schlieren images (Fig. 8). Figure 13 presents two pressure-deflection plots that compare frozen chemistry with equilibrium chemistry using nitrous

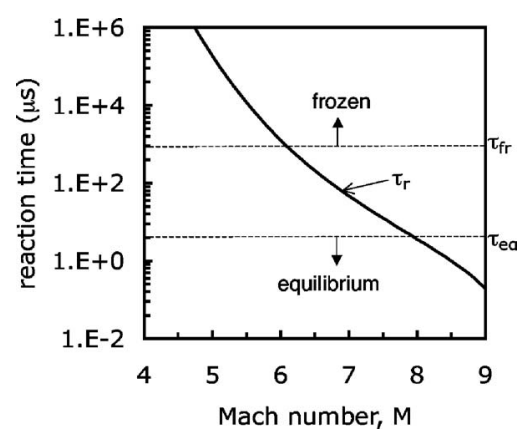

FIG. 12. The chemical dissociation reaction time of nitrous oxide as a function of Mach number Ma. The particle residence time $\tau_{r}$, equilibrium time threshold $\tau_{\text {eq }}$, and frozen threshold $\tau_{\text {fr }}$, are shown. 


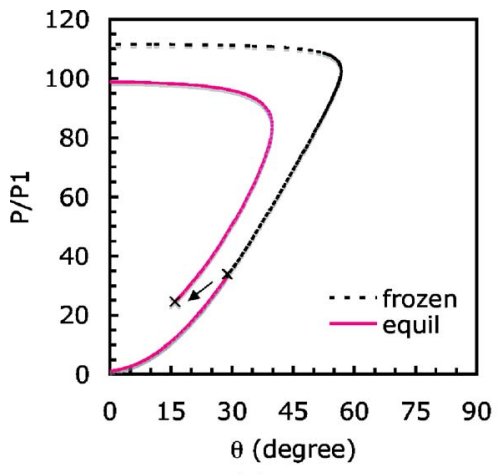

(a)

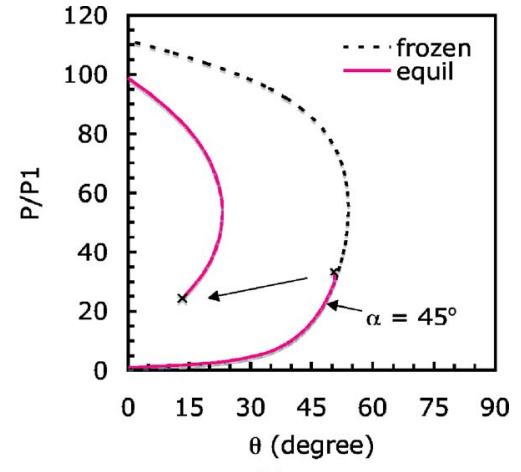

(b)
FIG. 13. Oblique shock normalized pressure $P / P_{1}$ as a function of flow deflection angle $\theta$ plotted for nitrous oxide with equilibrium and frozen composition. (a) Varying shock angle $\beta$ with $U=V_{\mathrm{CJ}}$. (b) Varying shock angle $\beta$, with $U$ obtained by Eq. (10). oxide as the test gas. In Fig. 13(a), the incident velocity is $U=2622 \mathrm{~m} / \mathrm{s}$, which corresponds to the detonation velocity of a $\Phi=2.5$ ethylene-oxygen mixture. The shock angle is varied from $\beta=\sin ^{-1}\left(c_{1} / U\right)$ to $\beta=90^{\circ}$. The equilibrium curve shows that at small shock angles, the reaction time is large, resulting in a frozen composition. As the angle $\beta$ increases, the nitrous oxide dissociates, denoted by the arrow from the frozen curve to the equilibrium curve. One important observation is that the equilibrium pressure-deflection curve lies inside the frozen curve. This is opposite to what occurs in endothermic dissociation with oxygen or nitrogen. ${ }^{6}$ Figure 13(b) is a pressure deflection curve calculated by varying the interface angle $\alpha$, thus fixing the shock angle $\beta$ and the incident inflow Mach number using the detonation-refraction analysis and Eq. (10), respectively.

When the contact surface angle $\alpha=45^{\circ}$, the post-shock solution is shown to lie on the frozen pressure deflection curve since the transmitted shock $\left(\mathrm{Ma}_{n}=3.9\right)$ has a decomposition reaction time larger than $\tau_{\mathrm{fr}}$. This is consistent with the images obtained from experiments \#1916, 1917, and 1921, where immediately behind the dark line corresponding to the transmitted shock there is a light section that ends at the leading edge of the darkly shaded turbulent mixing zone. The Mach stem in the case with a nitrous oxide test gas [Fig. $8(\mathrm{c})$ ] is slightly curved and is measured to move at a mean velocity of $V_{\mathrm{CJ}}=2622 \mathrm{~m} / \mathrm{s}$. At this velocity, the post-shock state behind the Mach stem is most accurately represented using equilibrium chemistry. Experimentally the Mach stems are thick dark lines, possibly indicating the decomposition of nitrous oxide. The exothermicity of the nitrous oxide enhances the role of the Mach stem because it increases the normal component of the post-shock velocity and therefore reduces the maximum flow deflection angle shown in Fig. 13(a). The CJ detonation velocity was calculated to be $V_{\mathrm{CJ}}=1691 \mathrm{~m} / \mathrm{s}$ for nitrous oxide decomposition. Thus the Mach stem velocity corresponds to a nitrous oxide detonation that is overdriven by a factor of 1.55 .

\section{B. Shear layer growth}

The turbulent mixing zone visible in sharp interface experiments (Fig. 1) forms as a result of a mismatch in flow velocity at the post-detonation contact surface $\mathrm{CS}^{\prime}$. Understanding the growth, entrainment of fluid, and subsequent mixing is essential in assessing the TMZ impact on the over- all flow structure and the extent to which secondary combustion occurs. A vast amount of research has been carried out in the field of turbulent shear layers, ${ }^{19,20}$ which is used to guide the following discussion. The analysis in this section estimates the growth angle of the shear layer and the entrainment that results from both free streams. In order to make use of the existing theories and models it is essential to characterize the key parameters involved in shear layer formation.

\section{Shear layer classification}

Shear layer entrainment and growth is highly dependent on the free stream flow conditions and free stream thermodynamic states. The Reynolds number defined as

$$
\operatorname{Re}=\frac{\delta_{G} \Delta U}{\nu}
$$

plays an important role in classifying the type of mixing and growth that results. $\delta_{G}, \Delta U$, and $\nu$ represent the local shear layer thickness, the difference in velocity of the two streams, and the kinematic viscosity, respectively. $\mathrm{Re} \approx 10^{4}$ corresponds to the critical Reynolds number above which a transition to intense molecular mixing occurs in the shear layer. $^{21,22}$ The transition to a turbulent mixing layer results in visibly different shear layer behavior. The value of Re for the current experimental data was calculated over the length of the shear layer and found to be equal to the transition Re for shear layer thicknesses, $\delta_{G} \approx 1-2 \mathrm{~mm}$. Practically, this means that the shear layers are always turbulent and involve intense molecular mixing. Calculations of the Schmidt number yield $\mathrm{Sc} \approx 1$ over the range of parameters in the sharp interface experiments. It is convenient to analyze the shear layer in a reference frame convecting with velocity $U_{c}$ that is in the range

$$
U_{2}<U_{c}<U_{1}
$$

where $U_{1}$ and $U_{2}$ are the velocities of the fast and slow streams, respectively, shown in Fig. 1. The value of $U_{c}$ for incompressible flow is determined ${ }^{19}$ to be 


$$
\frac{U_{c}}{U_{1}} \approx \frac{1+r \sqrt{s}}{1+\sqrt{s}}
$$

using the continuity and momentum equations under the condition that the pressures on either side of the shear layer are equal. The parameters $r=U_{2} / U_{1}$ and $s=\rho_{2} / \rho_{1}$ relate the velocity and density of the top and bottom streams. In the current experiments the velocities $U_{1}$ and $U_{2}$ are obtained using the detonation refraction analysis. $U_{1}$ corresponds to the velocity of the partially oxidized detonation products, and $U_{2}$ is the velocity of the shocked test gas. The velocities $\vec{w}_{3}, \vec{w}_{4}$, and $\vec{w}_{1}$ and the contact surface deflection angle $\delta$ in the nodefixed reference frame are used to specify

$$
\begin{aligned}
& U_{1}=\left|\vec{w}_{3}\right|-\left|\vec{w}_{1}\right| \cos (\delta-\alpha-\pi), \\
& U_{2}=\left|\vec{w}_{4}\right|-\left|\vec{w}_{1}\right| \cos (\delta-\alpha-\pi) .
\end{aligned}
$$

It is convenient to define convective Mach numbers,

$$
\begin{aligned}
& \mathrm{Ma}_{c 1}=\frac{U_{1}-U_{c}}{c_{1}}, \\
& \mathrm{Ma}_{c 2}=\frac{U_{c}-U_{2}}{c_{2}},
\end{aligned}
$$

to gauge the compressibility of the two streams. Here, $\mathrm{Ma}_{c 1}$ and $\mathrm{Ma}_{c 2}$ correspond to $U_{1}$ and $U_{2}$, respectively, and $c_{1}$ and $c_{2}$ are the local sound speeds of each stream. It was observed $^{23}$ that the growth rate of a shear layer decreases with increasing free stream Mach number. The influence of compressibility appears to be minimal with the largest convective Mach numbers found in the nitrous oxide mixtures on the order of $\mathrm{Ma}_{c}=0.18$. The convective flow is considered subsonic in all cases.

The shear layer thickness $\delta_{G}$, scales with the coordinate $x$, defined as the downstream distance measured from the point of first contact of the two streams (Fig. 1). The growth rate of the shear layer, i.e., $\delta_{G} / x$, can be expressed ${ }^{19}$ as a function of the density ratio and velocity ratio to be

$$
\frac{\delta_{G}}{x}(r, s) \approx C_{\delta} \frac{(1-r)(1+\sqrt{s})}{2(1+r \sqrt{s})}\left(1-\frac{(1-\sqrt{s}) /(1+\sqrt{s})}{1+2.9(1+r) /(1-r)}\right),
$$

for shear layers where the convective Mach number of both streams, $\mathrm{Ma}_{c} \approx 0$. The constant $C_{\delta}$ was determined ${ }^{23}$ to lie between the values

$$
0.25<C_{\delta}<0.45
$$

The range in $C_{\delta}$ is attributed to the role that the initial flow conditions play on the shear layer growth. ${ }^{19}$

The growth rates are corrected for compressibility ${ }^{24}$ by the functional relationship

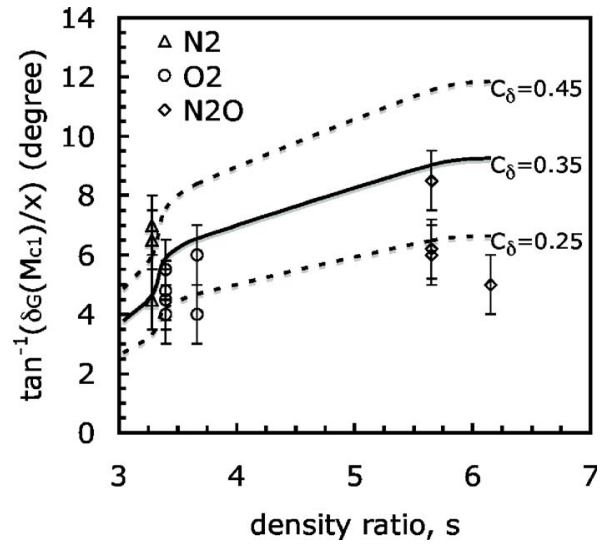

FIG. 14. The experimental and calculated Eq. (16) growth angle plotted as a function of density ratio.

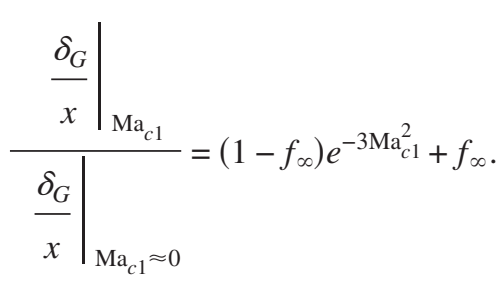

Equation (18) relates the ratio of the compressible shear layer with convective Mach number $\mathrm{Ma}_{c 1}$ to the growth rate of an equivalent system with $\mathrm{Ma}_{c 1} \approx 0$ as a function of $\mathrm{Ma}_{c 1}$. The asymptotic value of the constant was determined to be $f_{\infty}=0.2$.

It is now a question of relating the computed values to those measured in the experiments. The difficulty lies in choosing the appropriate value of the coefficient $C_{\delta}$. The shear layer growth angle $\Theta_{\text {exp }}$, is measured for each experiment and reported in Table I. Each measurement has an uncertainty of $1^{\circ}$. The angle $\Theta_{\text {gap }}$ is listed and defined as the angle between the transmitted shock and the leading edge of the TMZ (Fig. 1).

The experimentally measured growth angle $\Theta_{\text {exp }}$ is plotted as a function of density ratio $s$ in Fig. 14, with data corresponding to the experiments listed in Table I. For comparison the predicted growth rate angle is shown by the solid line with $C_{\delta}=0.35$. Upper and lower bounds of the calculation are included in the figure to illustrate the range of possible values by varying $C_{\delta}$ from 0.25 (lower curve) to 0.45 (upper curve). The experiment and theory are consistent for low density ratios where either nitrogen or oxygen was used as the test gas. The experimental results for large density ratio are lower than the predicted growth angle.

The dependence of $C_{\delta}$ on initial conditions adds complexity when trying to estimate the growth rate. A methodology was developed ${ }^{25}$ to calculate $C_{\delta}$ using the ratio of the experimental growth rate to the compressibility-corrected growth rate normalized by the unknown coefficient $C_{\delta}$, with results shown in Table IV. As shown, $C_{\delta}$ ranges over the previously observed values $0.25<C_{\delta}<0.45$ depending on the initial conditions.

The volumetric entrainment ratio $E_{v}$ relates the entrainment of high-speed fluid to the low-speed fluid. It was 
TABLE IV. Table comparing the calculated and experimentally averaged growth angles $\Theta_{\text {model }}$ and $\Theta_{\exp }$ for ethylene-oxygen mixtures, with equivalence ratio $\Phi$, and oxygen, nitrogen, or nitrous oxide as the test gas. The convective Mach numbers, velocity ratio, density ratio, and entrainment ratio, $E_{v}$, are listed.

\begin{tabular}{lcccccccccc}
\hline \hline Test gas & $\Phi$ & $r$ & $s$ & $\mathrm{Ma}_{c 1}$ & $\mathrm{Ma}_{c 2}$ & $U_{c}(\mathrm{~m} / \mathrm{s})$ & $\Theta_{\text {model }}$ & $\Theta_{\exp }$ & $E_{v}$ & $C_{\delta}$ \\
\hline $\mathrm{O}_{2}$ & 2.5 & 0.45 & 3.67 & 0.13 & 0.04 & 183.3 & 6.6 & 5.3 & 2.3 & 0.28 \\
$\mathrm{~N}_{2}$ & 2.5 & 0.60 & 3.28 & 0.08 & 0.02 & 178.9 & 4.7 & 6.0 & 2.1 & 0.45 \\
$\mathrm{~N}_{2} \mathrm{O}$ & 2.5 & 0.21 & 6.15 & 0.36 & 0.06 & 161.4 & 9.3 & 5.0 & 3.0 & 0.19 \\
$\mathrm{O}_{2}$ & 1.0 & 0.50 & 3.40 & 0.11 & 0.03 & 166.7 & 5.9 & 4.7 & 2.2 & 0.28 \\
$\mathrm{~N}_{2}$ & 1.0 & 0.66 & 3.04 & 0.06 & 0.02 & 163.1 & 3.8 & & 2.0 & \\
$\mathrm{~N}_{2} \mathrm{O}$ & 1.0 & 0.23 & 5.65 & 0.32 & 0.06 & 147.1 & 9.0 & 6.9 & 2.9 & 0.27 \\
\hline \hline
\end{tabular}

observed $^{21}$ that the two streams do not contribute equally to the shear layer, and a model was proposed ${ }^{26}$ for the entrainment ratio,

$$
E_{v}=\frac{U_{1}-U_{c}}{U_{c}-U_{2}}\left(1+C_{l} \frac{1-r}{1+r}\right) .
$$

The value of coefficient $C_{l}$ is approximately equal to 0.68 . Table IV gives the entrainment ratio values computed using Eq. (19) and corrected for compressibility ${ }^{27}$ over the range of initial conditions that are important when considering combustion and subsequent energy release in the shear layer. The entrainment ratio of the partially oxidized detonation products and test gas in the shear layer is around 2-2.5.

The effect of chemical reactions inside the shear layer has been shown ${ }^{16}$ to decrease the growth rate and consequently, the volumetric entrainment, linearly as a function of the heat release. This is because the expansion due to the heat release reduces the entrainment requirements of the shear layer. Experiments with different equivalence ratios (Table I) were carried out, and it was found that the shear layer growth angle decreases when nitrous oxide is used as the test gas while the growth angle does not change for oxygen as the test gas.

The Damköhler number

$$
\mathrm{Da} \equiv \frac{\tau_{m}}{\tau_{i}}
$$

is the ratio of the mixing time scale $\tau_{m}$ to the chemical time scale $\tau_{i}$. The chemical reaction time scale, calculated using the homogeneous mixing ignition time, is on the order of $1 \mu$ s for partially oxidized combustion products mixed with oxygen or nitrous oxide. The calculations are carried out with $\alpha=45^{\circ}$, which is consistent with the experiments. $\tau_{m}$ is estimated using the ratio of the downstream distance $x$, and the convective entrainment velocity $U_{c}-U_{2}$. The mixing time is on the order of $1 \mathrm{~ms}$ using the half-height of the GDT as a value for $x$, which results in $\mathrm{Da} \approx 1000$. The high Damköhler number for the conditions in the experiments indicates that the chemical reaction rate is much faster than the mixing rate meaning that the "fast" chemistry limit is relevant in most cases. This means that as soon as fluid from the two streams are molecularly mixed, the chemical reactions proceed to form products determined by the local equivalence ratio. The limit of fast chemistry results in $\delta_{p}<\delta_{m}$, where $\delta_{p}$ is the thickness of the region where chemical reactions have reached completion. Since $\delta_{p}$ depends on the local equivalence ratio, a function of position in the shear layer, the total region where chemical reactions have reached completion will always be less than $\delta_{m}$. For gas phase flows ${ }^{19}$ with $\operatorname{Re}>10^{4}$, results in the ratio

$$
\frac{\delta_{m}}{\delta_{G}}=0.49 .
$$

The ramification of Eq. (21) is that only half of the visible turbulent shear layer is mixed on a molecular level which can then react to form products based on the local mixture composition.

\section{SUMMARY}

Detonation refraction experiments were carried out that used a nitro-cellulose membrane to separate an ethyleneoxygen mixture from either oxygen, nitrogen, or nitrous oxide. The experimentally measured wave angles were found to be consistently a couple of degrees less than the prediction of the detonation refraction analysis. The choice of combustible mixture allowed for secondary combustion to occur in the TMZ. Mixture equivalence ratios of 1 and 2.5 were tested and determined to have minimal impact on the wave features observed.

A Mach stem formed in all experiments because the transmitted oblique shocked fluid could not be deflected back tangent to the wall with a reflected shock wave. The enhanced Mach stem when nitrous oxide was used as the test gas is attributed to the exothermic decomposition that occurs behind the normal shock that is visible in the experimental images as a thick dark Mach stem compared to cases with oxygen and nitrogen. Both oxygen and nitrogen are endothermic, and within the time scales of this analysis the composition of the test gas remains frozen.

Analysis of the shear layer growth, mixing, and ignition process revealed that the combustion rate is limited by the rate of molecular mixing. In addition, the thickness of the mixing region is estimated to be only half the visible thickness of the turbulent shear layer. The experimentally measured shear layer growth angles were found to agree with the calculated values within the range of accepted values of the 
constant $C_{\delta}$. Although the role of the shear layer in the detonation refraction analysis has not been formally addressed in this study, it is anticipated that the matching conditions at the contact surface would need to include the entrainment requirements of the shear layer.

${ }^{1}$ D. H. Lieberman and J. E. Shepherd, "Detonation interaction with a diffuse interface and subsequent chemical reaction," Shock Waves 16, 421 (2007).

${ }^{2}$ L. G. Gvozdeva, "Refraction of detonation waves incident on boundary between 2 gas mixtures," Sov. Phys. Tech. Phys. 6, 527 (1961).

${ }^{3}$ E. K. Dabora, D. Desbores, C. Guerraud, and H. G. Wagner, "Oblique detonations at hypersonic velocities," Prog. Astronaut. Aeronaut. 133, 187 (1991).

${ }^{4}$ N. A. Tonello, M. Sichel, and C. W. Kauffman, "Mechanisms of detonation transmission in layered $\mathrm{H}_{2}-\mathrm{O}_{2}$ mixtures," Shock Waves 5, 225 (1995).

${ }^{5}$ R. Samtaney and D. I. Pullin, "Self-similar hypervelocity shock interactions with oblique contact discontinuities," Shock Waves 8, 299 (1998).

${ }^{6}$ S. R. Sanderson, H. G. Hornung, and B. Sturtevant, "Aspects of planar, oblique and interacting shock waves in an ideal dissociating gas," Phys. Fluids 15, 1638 (2003).

${ }^{7}$ E. K. Dabora, J. A. Nicholls, and R. B. Morrison, "The influence of a compressible boundary on the propagation of gaseous detonations," Tenth (International) Symposium on Combustion, University of Cambridge, August 17-21, 1964 (Combustion Institute, Pittsburgh 1965), pp. 817-830.

${ }^{8}$ R. Akbar, "Mach reflection of gaseous detonations," Ph.D. thesis, California Institute of Technology, Pasadena, California, August 1997.

${ }^{9}$ J. Austin, "The role of instability in gaseous detonation," Ph.D. thesis, California Institute of Technology, Pasadena, California, June 2003.

${ }^{10}$ D. R. White, "Turbulent structure of gaseous detonations," Phys. Fluids 4, 465 (1961).

${ }^{11}$ S. Kumar, "An experimental investigation of Richtmyer-Meshkov instability," Ph.D. thesis, California Institute of Technology, 2002.

${ }^{12}$ M. Brouillette, "The Richtmyer-Meshkov instability," Annu. Rev. Fluid Mech. 34, 445 (2002).
${ }^{13}$ D. Lieberman, "Detonation interaction with sharp and diffuse interfaces," Ph.D. thesis, California Institute of Technology, Pasadena, California, November 2005.

${ }^{14}$ L. F. Henderson, "On the refraction of shock waves," J. Fluid Mech. 198, 365 (1989).

${ }^{15}$ P. A. Thompson, Compressible-Fluid Dynamics, Advanced Engineering Series (McGraw-Hill, New York, 1988).

${ }^{16}$ J. C. Hermanson and P. E. Dimotakis, "Effects of heat release in a turbulent, reacting shear layer," J. Fluid Mech. 199, 333 (1989).

${ }^{17}$ H. G. Hornung, "Regular and mach reflection of shock waves," Annu. Rev. Fluid Mech. 18, 33 (1986).

${ }^{18}$ H. G. Hornung, H. Oertel, and R. J. Sandeman, "Transition to mach reflexion of shock waves in steady and pseudosteady flow with and without relaxation," J. Fluid Mech. 90, 541 (1979).

${ }^{19}$ P. E. Dimotakis, Turbulent Free Shear Layer Mixing and Combustion (American Institute of Aeronautics and Astronautics, Reston, VA, 1991), Chap. 5, pp. 265-340

${ }^{20}$ P. E. Dimotakis, "Turbulent mixing," Annu. Rev. Fluid Mech. 37, 329 (2005).

${ }^{21}$ J. H. Konrad, "An experimental investigation of mixing in twodimensional turbulent shear flows with applications to diffusion-limited chemical reactions," Ph.D. thesis, California Institute of Technology, 1976.

${ }^{22}$ M. M. Koochesfahani and P. E. Dimotakis, "Mixing and chemical reactions in a turbulent liquid mixing layer," J. Fluid Mech. 170, 83 (1986).

${ }^{23}$ G. L. Brown and A. Roshko, "On density effects and large structure in turbulent mixing layers," J. Fluid Mech. 64, 775 (1974).

${ }^{24}$ D. Papamoschou and A. Roshko, "The compressible turbulent shear layer: An experimental study," J. Fluid Mech. 197, 453 (1988).

${ }^{25}$ M. D. Slessor, M. Zhuang, and P. E. Dimotakis, "Turbulent shear-layer mixing: Growth-rate compressibility scaling," J. Fluid Mech. 414, 35 (2000).

${ }^{26}$ P. E. Dimotakis, "Two-dimensional shear-layer entrainment," AIAA J. 24, 1791 (1986).

${ }^{27}$ J. L. Hall, P. E. Dimotakis, and H. Rosemann, "Experiments in nonreacting compressible shear layers," AIAA J. 31, 2247 (1993). 